\title{
Observation of Parity Nonconservation in Møller Scattering
}

P. L. Anthony, ${ }^{7}$ R. G. Arnold,${ }^{3}$ C. Arroyo,${ }^{3}$ K. Baird, ${ }^{7}$ K. Bega,${ }^{2}$ J. Biesiada,,${ }^{1,4}$ P. E. Bosted, ${ }^{3}$ M. Breuer, ${ }^{3}$ R. Carr, ${ }^{2}$ G. D. Cates, ${ }^{10}$ J-P. Chen, ${ }^{9}$ E. Chudakov, ${ }^{9}$ M. Cooke, ${ }^{1}$ F. J. Decker, ${ }^{7}$ P. Decowski, ${ }^{6}$ A. Deur, ${ }^{10}$ W. Emam, ${ }^{8}$ R. Erickson, ${ }^{7}$ T. Fieguth, ${ }^{7}$ C. Field, ${ }^{7}$ J. Gao, ${ }^{2}$ K. Gustafsson, ${ }^{2, *}$ R. S. Hicks, ${ }^{3}$ R. Holmes, ${ }^{8}$ E. W. Hughes, ${ }^{2}$ T. B. Humensky, ${ }^{4}$ G. M. Jones, ${ }^{2}$ L. J. Kaufman, ${ }^{3}$ Yu. G. Kolomensky, ${ }^{1}$ K. S. Kumar, ${ }^{3}$ D. Lhuillier, ${ }^{5}$ R. Lombard-Nelsen, ${ }^{5}$ P. Mastromarino, ${ }^{2}$ B. Mayer, ${ }^{5}$ R. D. McKeown, ${ }^{2}$ R. Michaels, ${ }^{9}$ M. Olson, ${ }^{7}$ K. D. Paschke, ${ }^{3}$ G. A. Peterson, ${ }^{3}$ R. Pitthan, ${ }^{7}$ K. Pope,${ }^{6,}$ D. Relyea, ${ }^{4,7}$ S. E. Rock,${ }^{3}$ O. Saxton, ${ }^{7}$ G. Shapiro,,${ }^{1,}$ J. Singh, ${ }^{10}$ P. A. Souder,${ }^{8}$ Z. M. Szalata, ${ }^{7}$ W. A. Tobias, ${ }^{10}$ B. T. Tonguc, ${ }^{8}$ J. Turner, ${ }^{7}$ B. Tweedie, ${ }^{1}$ A. Vacheret, ${ }^{5}$ D. Walz, ${ }^{7}$ T. Weber, ${ }^{7}$ J. Weisend, ${ }^{7}$ D. Whittum, ${ }^{7}$ M. Woods ${ }^{7}$ and I. Younus ${ }^{8}$

\section{(SLAC E158 Collaboration)}

\author{
${ }^{1}$ University of California, Berkeley, California 94720, USA \\ ${ }^{2}$ California Institute of Technology, Pasadena, California 91125, USA \\ ${ }^{3}$ University of Massachusetts, Amherst, Massachusetts 01003, USA \\ ${ }^{4}$ Princeton University, Princeton, New Jersey 08544, USA \\ ${ }^{5}$ CEA Saclay, DAPNIA/SPhN, F-91191 Gif-sur-Yvette, France \\ ${ }^{6}$ Smith College, Northampton, Massachusetts 01063, USA \\ ${ }^{7}$ Stanford Linear Accelerator Center, Menlo Park, California 94025, USA \\ ${ }^{8}$ Syracuse University, Syracuse, New York 13244, USA \\ ${ }^{9}$ Thomas Jefferson Laboratory, Newport News, Virginia 23606, USA \\ ${ }^{10}$ University of Virginia, Charlottesville, Virginia 22903, USA
}

(Received 11 December 2003; published 7 May 2004)

\begin{abstract}
We report a measurement of the parity-violating asymmetry in fixed target electron-electron (Møller) scattering: $A_{\mathrm{PV}}=[-175 \pm 30$ (stat) \pm 20 (syst) $] \times 10^{-9}$. This first direct observation of parity nonconservation in Møller scattering leads to a measurement of the electron's weak charge at low energy $Q_{W}^{e}=-0.053 \pm 0.011$. This is consistent with the standard model expectation at the current level of precision: $\sin ^{2} \theta_{\mathrm{W}}\left(M_{Z}\right)_{\overline{\mathrm{MS}}}=0.2293 \pm 0.0024$ (stat) \pm 0.0016 (syst) \pm 0.0006 (theory).
\end{abstract}

DOI: 10.1103/PhysRevLett.92.181602

Precision measurements of weak neutral current (WNC) processes mediated by $Z^{0}$ exchange stringently test the standard model of electroweak interactions. While most WNC measurements have been performed at high energy colliders, the comprehensive search for new physics at $\mathrm{TeV}$ energies also requires precision measurements at low momentum transfer $\left(Q^{2} \ll M_{Z}^{2}\right)$.

One class of such measurements involves the scattering of longitudinally polarized electrons from unpolarized targets, allowing the determination of a parity-violating asymmetry $A_{\mathrm{PV}} \equiv\left(\sigma_{R}-\sigma_{L}\right) /\left(\sigma_{R}+\sigma_{L}\right)$, where $\sigma_{R(L)}$ is the cross section for incident right(left)-handed electrons. $A_{\mathrm{PV}}$ arises from the interference of the weak and electromagnetic amplitudes [1] and is sensitive to WNC coupling constants and thus the weak mixing angle $\theta_{\mathrm{W}}$.

The first observation of $A_{\mathrm{PV}}$ was made at the Stanford Linear Accelerator Center (SLAC) using a deuteron target [2]. That experiment established the basic experimental technique to determine small asymmetries that typically range from 0.1 to 100 parts per million (ppm). Subsequent measurements yielded improved precision and accuracy $[3,4]$. However, theoretical uncertainties related to the use of hadronic targets restricted the interpretation of the experimental results at the quantum loop level.
PACS numbers: 11.30.Er, 12.15.Mm, 13.66.Jn, 13.88.+e

In this Letter we report the first observation of $A_{\mathrm{PV}}$ in electron-electron (Møller) scattering. This purely leptonic reaction has a large cross section and has little theoretical uncertainty. The development of the $50 \mathrm{GeV}$ electron beam in SLAC End Station A (ESA) made possible a measurement of $A_{\mathrm{PV}}$ [5] with a precision that tests electroweak radiative corrections and probes physics beyond the standard model at the $\mathrm{TeV}$ scale.

At $50 \mathrm{GeV}$ and a center-of-mass scattering angle of $90^{\circ}$, $A_{\mathrm{PV}}$ in Møller scattering is predicted to be $\simeq 320$ parts per billion (ppb) [6] at tree level. Electroweak radiative corrections $[7,8]$ and the experimental acceptance reduce the measured asymmetry by more than $50 \%$. The principal components of the experimental apparatus, designed to measure $A_{\mathrm{PV}}$ to better than $10 \%$, were the polarized electron beam, a liquid hydrogen target, a spectrometer/ collimator system, and detectors. Møller-scattered electrons were directed into a calorimeter by a magnetic spectrometer. The asymmetry was measured by extracting the fractional difference in the integrated calorimeter response for incident right- and left-handed beam pulses.

The longitudinally polarized electron beam, with up to $5.5 \times 10^{11}$ electrons in $\approx 270 \mathrm{~ns}$ pulses at $120 \mathrm{~Hz}$, was produced by optical pumping of a strained GaAs 
photocathode [9] by circularly polarized laser light [10]. The sign of the laser circular polarization state determined the electron beam helicity. The helicity sequence of the pulse train was made up of quadruplets consisting of two consecutive pulses with pseudorandomly chosen helicities, followed by their complements, yielding two independent right-left "pulse pairs" every $33 \mathrm{~ms}$.

Careful optimization of optical components [10] minimized intrinsic intensity and position differences between right- and left-helicity beams that resulted from imperfections in the laser light and the photocathode response. Additionally, helicity-dependent corrections were applied to the laser beam in a feedback loop using periodic average measurements of beam asymmetries. The beam intensity and position were measured at the upstream and downstream ends of the accelerator with typical accuracies per pulse pair of $50 \mathrm{ppm}$ in intensity, $50 \mathrm{ppm}$ in energy, and $2 \mu \mathrm{m}$ [11] in position. Cumulative beam asymmetries at the target were reduced to $<500 \mathrm{ppb}$ in intensity, $<10 \mathrm{ppb}$ in energy, and $<50 \mathrm{~nm}$ in position.

A schematic diagram of the apparatus in ESA is shown in Fig. 1. The 0.5 MWelectron beam first passed through a $1.57 \mathrm{~m}$ long cylindrical cell filled with liquid hydrogen [12]. The cell was part of a target loop consisting of a motor, impeller, and heat exchanger, which circulated the liquid hydrogen at $\approx 5 \mathrm{~m} / \mathrm{s}$. Aluminum meshes in the fluid path surrounding the electron beam enhanced turbulence and mixing. These features allowed the absorption of $\approx 500 \mathrm{~W}$ deposited by the beam while keeping density fluctuations below $40 \mathrm{ppm}$ per pulse pair.

Scattered particles with a laboratory scattering angle between 4.4 and $7.5 \mathrm{mr}$ over the full range of the azimuth were selected by the magnetic spectrometer [13] while the primary beam and forward angle photons passed unimpeded to the beam dump. Sixty meters downstream of the target, the charged particle flux was approximately azimuthally symmetric about the beam axis. Møller electrons in the range $13-24 \mathrm{GeV}$ formed a ring spatially separated from electrons scattered from target protons (ep scattering). This Møller ring contained $\approx 2 \times 10^{7}$ electrons per pulse.

A scanning detector system provided a complete radial and azimuthal map of the charged particle flux. Figure 2 compares the measured radial profile at one azimuthal angle with Monte Carlo simulations of Møller and $e p$ scattering. The $e p$ flux within the Møller ring was the dominant background, estimated to be $\simeq 8 \%$.

For the asymmetry measurement, the charged particle flux was intercepted by the primary calorimeter, a $25 \mathrm{~cm}$ long cylindrical structure with a $15(35) \mathrm{cm}$ inner (outer) radius. It was assembled by layering planes of flexible fused-silica fibers between elliptical copper plates so as to withstand a $100 \mathrm{Mrad}$ radiation dose. The fibers directed Cherenkov light to air light guides, each of which terminated into a shielded photomultiplier tube (PMT). The regions I, II, and III in Fig. 2 were covered by 30, 20, and 10 PMTs, respectively, providing radial and azimuthal segmentation.

The small contribution of neutral particles, such as photons and neutrons, to the calorimeter response was measured in calibration runs. The asymmetry from pions was measured by using ten quartz bars arranged in azimuthal symmetry behind the Møller detector and lead shielding. Eight ionization chambers arranged in $45^{\circ}$ azimuthal sections intercepted charged particles with $\theta_{\text {lab }} \approx 1 \mathrm{mr}$. This "luminosity" detector monitored target density fluctuations and provided a check of the null asymmetry expected at such small scattering angles.

The data sample, constituting a total flux of just over $10^{20}$ electrons on target, was collected at beam energies of 45.0 and $48.3 \mathrm{GeV}$. Because of $g-2$ precession as the beam traversed a $24.5^{\circ}$ bend after acceleration, the two beam energies corresponded to opposite orientations of longitudinal beam polarization in ESA. Roughly equal statistics were thus accumulated with opposite signs for the measured asymmetry, which suppressed many systematic effects. In addition, the state of a half-wave plate in the laser line was toggled every other day, passively reversing the sign of the electron beam polarization. This guarded against helicity-correlated electronics cross talk.

For each beam pulse at $120 \mathrm{~Hz}$, a distributed data acquisition (DAQ) system was triggered to collect data from the polarized source electronics and the digitized integrated response of the detectors and beam monitors with negligibly small electronic dead time. Alternate DAQ triggers fell into two $60 \mathrm{~Hz}$ fixed-phase "time slots." Within these time slots, right-left pulse pairs were formed for independent asymmetry analyses.

Loose requirements were imposed on beam quality and beam monitor linearity. However, no helicity-dependent cuts were applied, other than the demand that the beam intensity asymmetry measured by two independent monitors agreed to within $10^{-3}$ for each pulse pair. In total, $8.6 \times 10^{7}$ pulse pairs satisfied all selection criteria. The right-left asymmetry in the integrated detector response for each pulse pair was computed and then corrected for fluctuations in the beam trajectory.

To first order, six correlated beam parameters described the trajectory of a beam pulse: intensity, energy, and

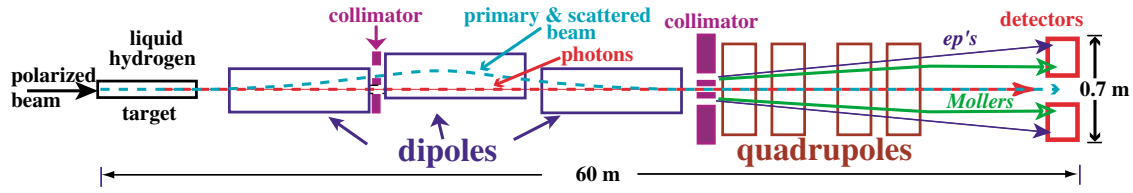

$181602-2$
FIG. 1 (color online). Schematic plan view of the experimental configuration in SLAC End Station A. 


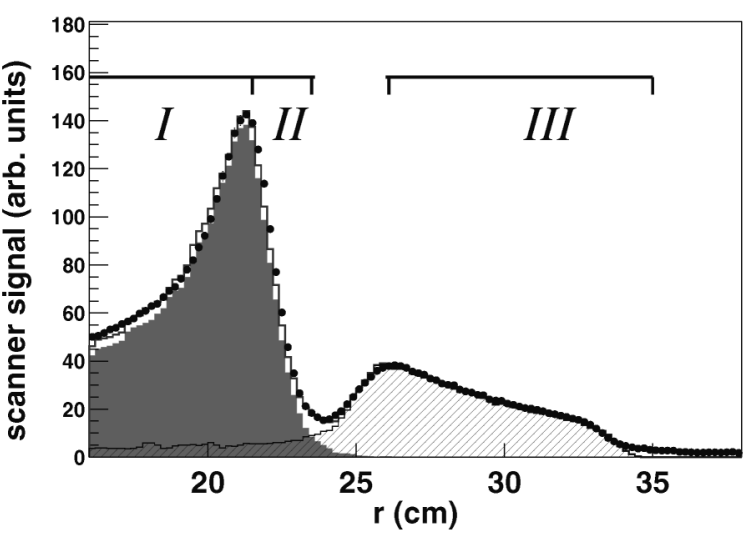

FIG. 2. The charged particle radial profile at the calorimeter. The points are the data scan, and the open histogram is the Monte Carlo simulation. Møller (shaded) and ep (hatched) contributions are also shown. Region I and III PMTs were used to measure Møller and ep asymmetries, respectively.

horizontal and vertical position and angle. Each beam parameter was measured by two independent monitors, such that device resolution and systematic effects could be studied.

Two methods were used to calibrate the detector sensitivity to each beam parameter and thus remove beaminduced random and systematic effects from the raw asymmetry. One method used a calibration subset of the pulses, where each beam parameter was modulated periodically around its average value by an amount large compared to nominal beam fluctuations with an $\sim 4 \%$ duty cycle. The other method applied an unbinned least squares linear regression to the pulses used for physics analysis. They yielded statistically consistent results to within $12 \mathrm{ppb}$. Final results were obtained with the latter, statistically more powerful technique.

The integrated responses of region $I$ PMTs (see Fig. 2) were averaged to form the raw asymmetry $A_{\text {raw }}$. Nearperfect azimuthal symmetry reduced the sensitivity to beam fluctuations and right-left asymmetries. The $A_{\text {raw }}$ pulse-pair distribution had an $\mathrm{rms}$ of $\approx 200 \mathrm{ppm}$. The cumulative beam asymmetry correction was $-41 \pm$ $3 \mathrm{ppb}$. A correction due to an azimuthal modulation of $A_{\text {raw }}$ [14] from a small nonzero transverse beam polarization component was found to be $-8 \pm 3 \mathrm{ppb}$.

Additional bias to $A_{\text {raw }}$ may arise from asymmetries in unmeasured beam quantities, such as higher order moments of beam distributions. Region II PMT channels were significantly more sensitive to such fluctuations and helped to limit possible bias in $A_{\text {raw }}$ to $10 \mathrm{ppb}$. Likewise, the luminosity detector was very sensitive to higher order effects, where the cumulative raw right-left asymmetry was $[-16 \pm 15$ (stat) $] \mathrm{ppb}$. This is consistent with the theoretical expectation, providing additional confirmation that higher order effects are under control. A separate study limited the bias due to beam spot-size fluctuations on $A_{\text {raw }}$ to $1 \mathrm{ppb}$, using data from a retractable wire array that was inserted into the beam during some of the data collection.

The physics asymmetry $A_{\text {phys }}$ was formed from $A_{\text {raw }}$ by correcting for background contributions, detector linearity, and beam polarization:

$$
A_{\text {phys }}=\frac{1}{P_{b} \epsilon} \frac{A_{\text {raw }}-\sum_{i} \Delta A_{i}}{1-\sum_{i} f_{i}} .
$$

$\Delta A_{i}$ and dilutions $f_{i}$ for various background sources are listed in Table I. The largest correction of $-26 \pm 6 \mathrm{ppb}$ was due to electrons from inelastic electron- and photonproton interactions. The measured asymmetry in region III PMTs was used as input, along with reasonable assumptions for the kinematic extrapolation to region I.

The electron beam polarization was $P_{b}=0.85 \pm 0.05$, measured every other day by a dedicated polarimeter using Møller scattering of beam electrons off a magnetized foil placed just upstream of the hydrogen target. The linearity of the calorimeter response was determined to be $\epsilon=0.99 \pm 0.01$.

Figure 3 shows $A_{\text {phys }}$ for all data, divided into 24 sequential samples. Each $A_{\text {phys }}$ measurement has sign reversals depending on the beam energy and the state of the half-wave plate. $A_{\mathrm{PV}}$ is obtained by correcting each result by the appropriate sign. The combined result is

$$
A_{\mathrm{PV}}=-175 \pm 30 \text { (stat) } \pm 20 \text { (syst) ppb, }
$$

establishing parity nonconservation in Møller scattering at the $5 \sigma$ level. $A_{\mathrm{PV}}$ is proportional to the product of the electron's vector and axial-vector neutral current couplings, parametrized as the weak charge $Q_{W}^{e}$ :

$A_{\mathrm{PV}}=\frac{G_{F} Q^{2}}{\sqrt{2} \pi \alpha} \frac{1-y}{1+y^{4}+(1-y)^{4}} \mathcal{F}_{\mathrm{b}} Q_{W}^{e} \equiv \mathcal{A}\left(Q^{2}, y\right) Q_{W}^{e}$,

where $G_{F}$ and $\alpha$ are the Fermi and fine structure constants, respectively [15], and $\mathcal{F}_{\mathrm{b}}=1.01 \pm 0.01$ accounted for kinematically weighted hard initial and final state radiation effects [16]. The effective analyzing power $\mathcal{A}=3.28 \pm 0.06 \mathrm{ppm}$ was determined from a

TABLE I. Corrections $\Delta A_{i}$ and dilutions $f_{i}$ to $A_{\text {raw }}$ and associated systematic uncertainties.

\begin{tabular}{crc}
\hline \hline Source & \multicolumn{1}{c}{$\Delta A(\mathrm{ppb})$} & $f$ \\
\hline Beam (first order) & $-41 \pm 3$ & \\
Beam (higher order) & $0 \pm 10$ & \\
Transverse polarization & $-8 \pm 3$ & \\
$e^{-}+p \rightarrow e^{-}+p(+\gamma)$ & $-8 \pm 2$ & $0.064 \pm 0.007$ \\
$e^{-}(\gamma)+p \rightarrow e^{-}+X$ & $-26 \pm 6$ & $0.011 \pm 0.003$ \\
High energy photons & $3 \pm 3$ & $0.004 \pm 0.002$ \\
Synchrotron photons & $0 \pm 5$ & $0.002 \pm 0.001$ \\
Neutrons & $-5 \pm 3$ & $0.003 \pm 0.001$ \\
Pions & $1 \pm 1$ & $0.001 \pm 0.001$ \\
\hline \hline
\end{tabular}




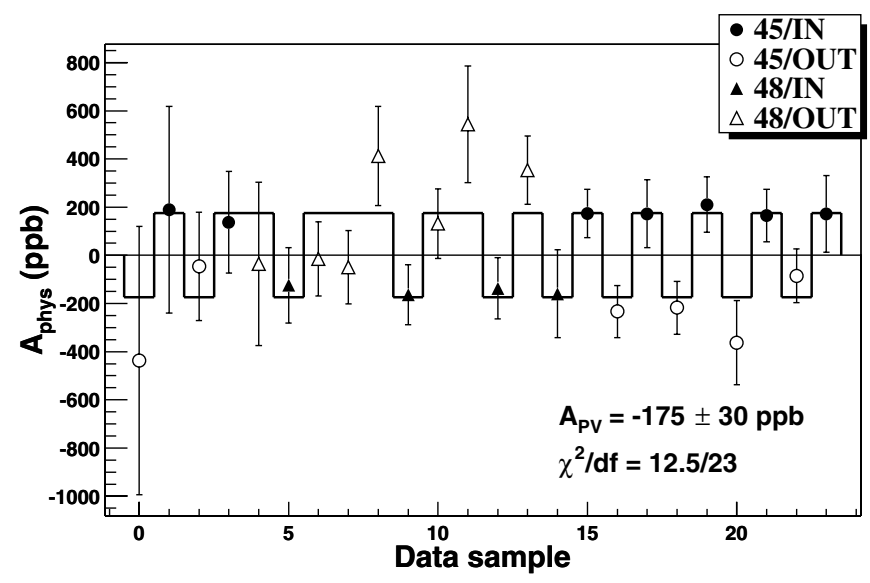

FIG. 3. $A_{\text {phys }}$ for each of 24 data samples. Data collected with half-wave plate inserted (removed) at a beam energy of 45 (48) $\mathrm{GeV}$ are shown as solid (open) circles (triangles). The solid line represents the grand average, with the expected modulation of the asymmetry sign for each beam energy and half-wave plate state. Only statistical uncertainties are shown.

Monte Carlo simulation that accounted for energy losses in the target and systematic uncertainties in the spectrometer setup. The average values of the kinematic variables were $Q^{2}=0.026(\mathrm{GeV} / c)^{2}$ and $y \equiv Q^{2} / s \simeq$ 0.6 , where $s$ is the square of the center-of-mass energy.

We find $Q_{W}^{e}=-0.053 \pm 0.009$ (stat) \pm 0.006 (syst), consistent with the standard model expectation $[7,15]$ of $-0.046 \pm 0.003$. As an example of the sensitivity of the measurement, the result can be used to limit the scale $\Lambda_{\mathrm{LL}}$ of a new left-handed contact interaction characterized by a term in the Lagrangian [17,18] $\mathcal{L}=\left(4 \pi / 2 \Lambda_{\mathrm{LL}}^{2}\right) \times$ $\left(\bar{e}_{L} \gamma_{\mu} e_{L}\right)$. At $95 \%$ C.L. a tree-level calculation yields $\Lambda_{\mathrm{LL}}^{+} \geq 7.2 \mathrm{TeV}$ and $\Lambda_{\mathrm{LL}}^{-} \geq 5.1 \mathrm{TeV}$, for potential positive and negative deviations, respectively.

In the context of the standard model, we find

$$
\begin{aligned}
\sin ^{2} \theta_{\mathrm{W}}\left(M_{\mathrm{Z}}\right)_{\overline{\mathrm{MS}}}= & 0.2293 \pm 0.0024(\text { stat }) \pm 0.0016 \text { (syst) } \\
& \pm 0.0006 \text { (theory) }
\end{aligned}
$$

The last error comes from the $Q^{2}$ evolution to the $Z$ pole.

The reported $A_{\mathrm{PV}}$ result is the most precise measurement of any asymmetry in electron scattering. The consistency of the result with the theoretical prediction provides significant new limits on $\mathrm{TeV}$ scale physics, comparable in sensitivity and complementary to other WNC measurements at low $Q^{2}$ [19]. Data from the first of three E158 run periods were used; the measurement accuracy is expected to improve by more than a factor of 2 when analysis of all the data is complete. The experimental techniques described in this Letter demonstrate the feasibility of measuring asymmetries with accuracies better than $10 \mathrm{ppb}$, applicable to new experiments under development [4].

We thank the SLAC staff for their efforts in helping develop and operate the E158 apparatus, and especially
A. Brachmann and T. Maruyama for their essential work on the polarized electron source. This work was supported by Department of Energy Contract No. DEAC03-76SF00515, and by the Division of Nuclear Physics at the Department of Energy and the Nuclear Physics Division of the National Science Foundation in the United States and the Commissariat à l'Énergie Atomique and the Centre National de la Recherche Scientifique in France.

*Present address: Helsinki Institute of Physics, Finland. ${ }^{\dagger}$ Deceased.

[1] Ya. B. Zel'dovich, Sov. Phys. JETP 94, 262 (1959).

[2] C. Y. Prescott et al., Phys. Lett. 84B, 524 (1979).

[3] W. Heil et al., Nucl. Phys. B327, 1 (1989); P. A. Souder et al., Phys. Rev. Lett. 65, 694 (1990); D. T. Spayde et al., Phys. Rev. Lett. 84, 1106 (2000); K. Aniol et al., Phys. Lett. B 509, 211 (2001).

[4] K. S. Kumar and P. A. Souder, Prog. Part. Nucl. Phys. 45, S333 (2000), and references therein.

[5] K. S. Kumar, R. S. Holmes, E.W. Hughes, and P. A. Souder, Mod. Phys. Lett. A 10, 2979 (1995); R. Carr et al., Stanford Linear Accelerator Center Proposal No. SLAC-E158, 1997 (unpublished).

[6] E. Derman and W. J. Marciano, Ann. Phys. (Berlin) 121, 147 (1979).

[7] A. Czarnecki and W. J. Marciano, Phys. Rev. D 53, 1066 (1996).

[8] A. Czarnecki and W. J. Marciano, Int. J. Mod. Phys. A 15, 2365 (2000); J. Erler, A. Kurylov, and M. J. RamseyMusolf, Phys. Rev. D 68, 016006 (2003); F. J. Petriello, Phys. Rev. D 67, 033006 (2003); A. Ferroglia, G. Ossola, and A. Sirlin, eprint hep-ph/0307200.

[9] T. Maruyama et al., Nucl. Instrum. Methods Phys. Res., Sect. A 492, 199 (2002).

[10] T. B. Humensky et al., e-print physics/0209067 [Nucl. Instrum. Methods Phys. Res. (to be published)].

[11] D. Whittum and Yu. Kolomensky, Rev. Sci. Instrum. 70, 2300 (1999).

[12] J. Gao et al., Nucl. Instrum. Methods Phys. Res., Sect. A 498, 90 (2003).

[13] D. Relyea, Ph.D. thesis, Princeton University, 2003 (unpublished).

[14] A. O. Barut and C. Fronsdal, Phys. Rev. 120, 1871 (1960); L. L. DeRaad, Jr. and Y. J. Ng, Phys. Rev. D 11, 1586 (1975).

[15] Particle Data Group, K. Hagiwara et al., Phys. Rev. D 66, 010001 (2002).

[16] V. Zykunov, Yad. Fiz. 66, annot. (2003).

[17] E. J. Eichten, K. D. Lane, and M. E. Peskin, Phys. Rev. Lett. 50, 811 (1983).

[18] M. J. Ramsey-Musolf, Phys. Rev. C 60, 015501 (1999).

[19] S. C. Bennett and C. E. Wieman, Phys. Rev. Lett. 82, 2484 (1999); G.P. Zeller et al., Phys. Rev. Lett. 88, 091802 (2002). 